\title{
BMJ Open Implementation of a complex intervention to improve hospital discharge: process evaluation of a cluster randomised controlled trial
}

\author{
Yael Rachamin (1D), Thomas Grischott (D) , Stefan Neuner-Jehle (D)
}

To cite: Rachamin $Y$, Grischott T, Neuner-Jehle S. Implementation of a complex intervention to improve hospital discharge: process evaluation of a cluster randomised controlled trial. BMJ Open 2021;11:e049872. doi:10.1136/ bmjopen-2021-049872

- Prepublication history and additional supplemental material for this paper are available online. To view these files, please visit the journal online (http://dx.doi.org/10.1136/ bmjopen-2021-049872).

Received 04 February 2021 Revised 31 March 2021 Accepted 23 April 2021
Check for updates

(c) Author(s) (or their employer(s)) 2021. Re-use permitted under CC BY-NC. No commercial re-use. See rights and permissions. Published by BMJ.

Institute of Primary Care, University of Zurich and University Hospital Zurich, Zurich, Switzerland

Correspondence to

Ms Yael Rachamin;

yael.rachamin@usz.ch

\section{ABSTRACT}

Objectives To study the implementation of a cluster randomised controlled effectiveness-implementation hybrid trial testing the effectiveness of a medication review at hospital discharge combined with a communication stimulus between hospital physicians (HPs) and general practitioners (GPs) on rehospitalisation of multimorbid older patients.

Design Extension of Grant's mixed method process evaluation framework to trials with multilevel clustering. Setting General internal medicine wards in Swiss hospitals.

Participants Convenience samples of 15 chief physicians (of 21 hospitals participating in the effectiveness trial), 60 (74) senior HPs, 65 (164) junior HPs and 187 (411) GPs. Implementation strategy Two-hour teaching sessions for senior HPs on a patient-centred, checklist-guided discharge routine.

Process evaluation components Data collection on recruitment, delivery and response from chief physicians (semistructured interviews), senior HPs, junior HPs, GPs (surveys) and patients (via HPs). Quantitative data were summarised using descriptive statistics, and interviews analysed using thematic analysis.

Outcome measures Intervention dose (quantitative), implementation fidelity (qualitative), feasibility and acceptability, facilitators and barriers, implementation support strategies.

Results Recruitment of hospitals was laborious but successful, with 21 hospitals recruited. Minimal workload and a perceived benefit for the clinic were crucial factors for participation. Intervention dose was high $(95 \%$ of checklist activities carried out), but intervention fidelity was limited (discharge letters) or unknown (medication review). Recruitment and retention of patients was challenging, partly due to patient characteristics (old, frail) and the COVID-19 pandemic: Only 612 of the anticipated 2100 patients were recruited, and $31 \%$ were lost to follow-up within the first month after discharge. The intervention was deemed feasible and helpful by HPs, and the relevance of the topic appreciated by both HPs and GPs.

Conclusions The results from this evaluation will support interpretation of the findings of the effectiveness study and may inform researchers and policy makers who aim at improving hospital discharge.

Trial registration number ISRCTN18427377.
Strengths and limitations of this study

- We used both quantitative and qualitative approaches to achieve an adequate insight into the implementation of the intervention.

- Our process evaluation was performed before instead of alongside or after the analysis and publication of the effectiveness findings, which is an innovative approach for process evaluations and ensures that the evaluation is blind to trial outcomes.

- Our application and extension of the framework described by Grant et al could serve as example and template for future implementation studies of complex multilevel interventions.

- The results from this evaluation will support interpretation of the findings of the effectiveness study and-positive results given-dissemination of our approach to further hospitals.

- The number of junior hospital physicians responding to the survey was limited by frequent rotations between and within hospitals.

\section{BACKGROUND}

Polypharmacy—often defined as the concomitant use of five or more medications-is associated with increased risk of adverse events, prescription errors, low patient adherence, morbidity, hospitalisation rates and mortality. ${ }^{1-4}$ During hospitalisation, patients are usually prescribed additional drugs while few drugs are deprescribed, so that the extent of polypharmacy is higher at discharge than at admission. ${ }^{5}$ Poor communication between healthcare providers after discharge additionally contributes to suboptimal prescribing. ${ }^{6}$

Previous systematic reviews provide some evidence that reducing polypharmacy improves health-related outcomes in older people, and that a variety of discharge planning interventions can reduce adverse events and healthcare utilisation in the post-discharge period. ${ }^{78}$ To our knowledge, no study has so far analysed the effects of a discharge strategy which incorporates both 
key aspects of deprescribing and collaborative communication between hospital physicians (HPs) and general practitioners (GPs) at hospital discharge. We therefore performed a two-armed cluster randomised controlled trial (RCT) investigating the effect of a medication review and improved information transfer at hospital discharge for patients aged 60 years or older with polypharmacy on rehospitalisation rates. ${ }^{9}$ The intervention was implemented via a teaching session and patient-centred checklists for HPs, and adaptations to the discharge letters. The complex intervention involved multiple sites, different levels of healthcare providers and different time points during the patients' hospital stay. A pragmatic approach allowed adapting the intervention to local conditions in the participating hospitals.

For complex RCTs, process evaluations are recommended to contextualise results. ${ }^{10}{ }^{11}$ It is often crucial to not only know whether but also when, why and how interventions 'work', particularly in the case of flexible and multisite interventions which may be implemented and received in different ways at the different sites. ${ }^{10} 12$ Various theoretical frameworks exist to guide the design and conduct of process evaluations. ${ }^{10}{ }^{13-15}$ For cluster RCTs specifically, Grant $e t \mathrm{al}^{16}$ developed a framework that considers the multilevel design with clusters and targeted individuals. We extended Grant's framework to evaluate the implementation of our trial with regard to recruitment of participants, intervention delivery, response of all parties involved and maintenance of the intervention.

The aim of our study was to provide information about process evaluation outcomes on different levels of the complex intervention in order to inform the interpretation of the effectiveness outcomes. The effectiveness outcomes will be published separately.

\section{METHODS}

\section{Design and setting}

This was a preplanned mixed method process evaluation of a cluster RCT involving patients (aged 60 years or older with five or more drugs prescribed) from 21 hospitals in the German-speaking part of Switzerland. The process evaluation, part of an effectiveness-implementation hybrid trial, ${ }^{17}$ was conducted in parallel to the main cluster RCT; effectiveness outcomes are still being collected and will be published separately. The study protocol for the full trial has been published elsewhere. ${ }^{9}$ The intervention was a patient-centred discharge procedure including critical medication review combined with a communication stimulus between HPs and GPs. The implementation strategy included (1) a 2-hour teaching session for senior HPs in internal medicine wards and rehabilitation hospitals who instructed junior HPs, (2) a checklist facilitating the medication review (online supplemental appendix 1) and (3) two adaptations to the discharge letter (reorganisation of the medication lists so that medication changes could easily be identified by the aftercare GP, and invitation to the GP to discuss potential changes of the medication plan). In the teaching session, senior HPs were presented some background evidence on the significance of multimorbidity and polypharmacy, and on age-dependent target values, and were then instructed on how to apply a simple medication review tool to the patients' medication lists ${ }^{1819}$ (see also checklist, online supplemental appendix $1)$. This was demonstrated on an example patient with

Intervention process (CRCT)

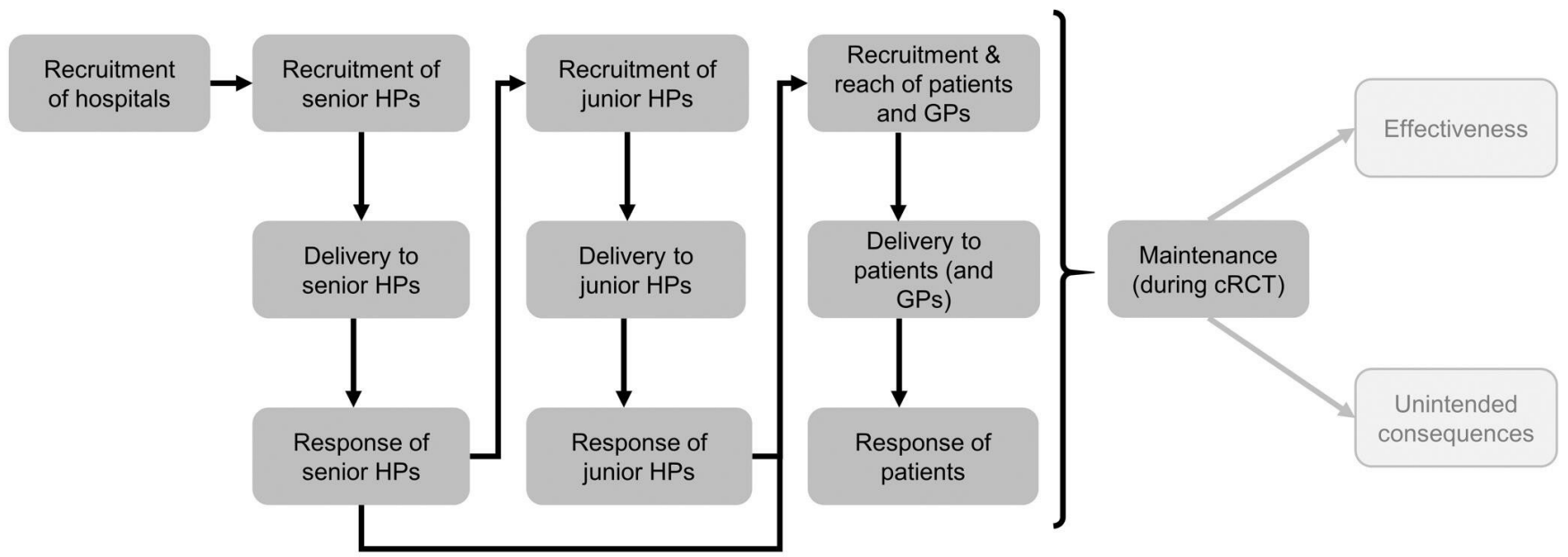

Theory: Empirical background for development of intervention strategy, interpretation of effects

Context: Wider context in which the trial is embedded; external (influencing) factors

Figure 1 Framework model for process (dark grey) and impact evaluation (light grey), adapted from Grant et al ${ }^{16}$. cRCT, cluster randomised controlled trial; GP, general practitioner; HP, hospital physician. 
multimorbidity and polypharmacy. Senior HPs were encouraged to engage in a discussion. In the second hour of the teaching session, data collection procedures were explained. In the control arm, senior HPs were given a 'sham' instruction (covering the significance of multimorbidity and polypharmacy, and explaining data collection procedures) and patients were discharged according to the usual discharge procedures as established in the individual hospitals.

Patients were followed up for 6 months beyond discharge for outcomes such as rehospitalisation, other physician contacts, current medication and quality of life, collected by questionnaires at 1, 3 and 6 months. After repeated requests for pending answers, we contacted the patients' relatives and/or GPs for complete follow-up data.

The process evaluation was based on a framework of Grant $e t a l^{16}$ which we then tailored to the specific multilevel nature of our intervention (figure 1). The original framework of Grant $e t a l^{16}$ distinguishes process elements (recruitment, delivery and response) of clusters and individuals from impact elements (effectiveness and unintended consequences). We added the levels 'hospitals' (the entities being recruited by the study team) and 'junior HPs' (who delivered the intervention to patients). We reported results in accordance with the Standards for Reporting Implementation Studies (StaRI) checklist. ${ }^{20}$

\section{Participants}

The recruitment procedure is described in the study protocol. ${ }^{9}$ We questioned chief physicians who decided about participation in the study, senior and junior HPs who were directly involved in the delivery of the intervention to patients, and GPs as downstream receivers of the intervention. Patients were not directly questioned. An overview of the flow of hospitals, senior HPs, junior HPs, GPs and patients through the study is shown in figure 2.

\section{Outcomes}

For the framework elements specified in figure 1, we aimed to explore and describe implementation along the following dimensions (where applicable):

- Intervention dose, that is, the quantity of the implemented intervention (eg, adherence rates).

- Implementation fidelity, that is, the quality of the implemented intervention compared with what was intended.

- Feasibility and acceptability, based on views and experiences of participants.

- Facilitators and barriers to implementation.

- Implementation support strategies to target facilitators, overcome barriers and ultimately improve implementation.

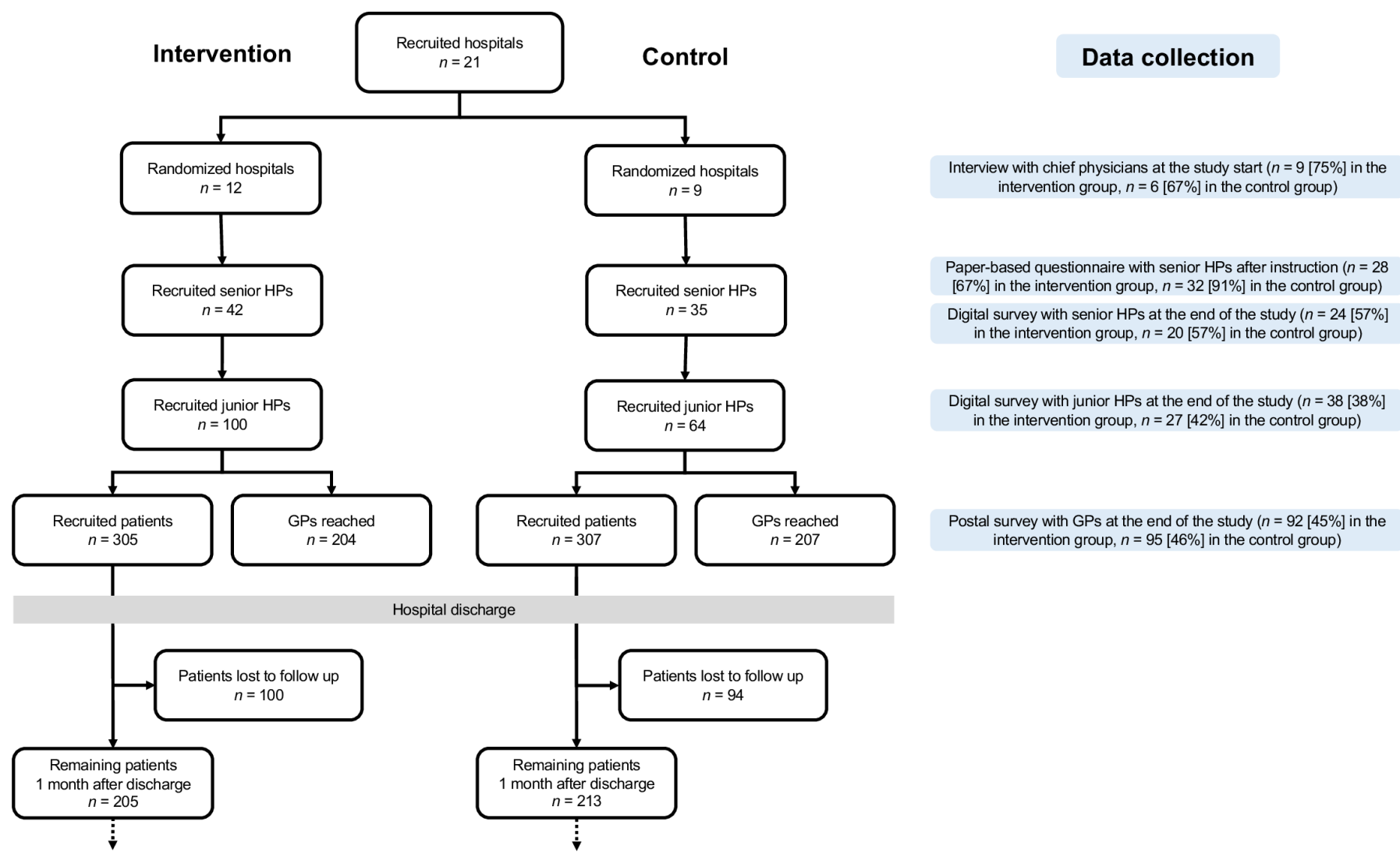

Figure 2 Flow of hospitals, senior hospital physicians (HPs), junior HPs, general practitioners (GPs) and patients through the study, by study arm. Blue boxes illustrate data collection tools with number of responses [response rates]. 


\section{Data collection}

We collected both qualitative and quantitative data (see online supplemental appendix 2 for the data collection tools). We conducted semistructured interviews with chief physicians at the beginning of the study, short paper-based surveys with senior HPs after instruction and an online survey at the end of the study with senior HPs and junior HPs. Both surveys had open-ended questions and quantitative parts. In addition to questions about feasibility, acceptability and awareness, the online survey contained two case vignettes with the intent of assessing knowledge transfer and increased awareness induced by the teaching session. The case vignettes described two model patients, and HPs were asked about their recommendations regarding the patients' medication. The case vignettes were pretested with three GPs at our institute and revised according to their responses. The fourth dedicated data collection tool was a short postal survey of GPs at the end of the study capturing their opinions regarding hospital discharge, with focus on the discharge letter, medication and contact. An overview of the data collection tools with response numbers and rates is incorporated in figure 2 .

In addition to these dedicated data collection tools, we used data from study instruments such as the patientspecific checklists (online supplemental appendix 1) where the intervening HPs had ticked off which parts of the intervention had actually been delivered (intervention dose). Finally, we recorded how chief physicians had initially planned to implement the intervention, and used our emails and protocols of phone calls with participants and patients.

\section{Data analysis}

Quantitative data were analysed with the R statistical software V.3.5.1 $1^{21}$ and Microsoft Excel (V.2016). We reported medians, interquartile ranges (IQRs), maxima (max) and minima ( $\min )$, or proportions ( $\%$ of non-missings) and numbers $(n)$, and compared groups using Wilcoxon and $\chi^{2}$ tests as appropriate. Significance was assumed for $p$ values $<0.05$. Likert scale items were dichotomised for text summaries.

The semistructured interviews were analysed by deductive thematic analysis, ${ }^{22}$ with a predefined focus on 'facilitators' and 'barriers' (to study participation). Two researchers (TG, SN-J) independently coded the 15 interviews until saturation (i.e., no further emergence of new codes) was reached, and subsequently grouped the codes into themes. A theme was accepted if listed by both of the two researchers, and similar themes on both the researchers' lists were merged by consensus. If there was disagreement between the two researchers, the third researcher (YR) operated as referee. Qualitative answers from the paper-based survey with senior HPs were also summarised according to the resulting themes.

\section{Patient and public involvement}

Patients were not involved in the planning of the study, but patient involvement is a core component of the medication review tool (see online supplemental appendix 1).

\section{RESULTS}

The results are presented along the elements specified in the framework (figure 1) and within each element further structured into (1) quantitative results, (2) qualitative results and (3) implementation strategies. The response rates for each data collection tool are given in figure 2 .

\section{Hospitals}

Recruitment

We approached 162 chief physicians of hospitals with a general internal medicine ward or of rehabilitation hospitals in German-speaking Switzerland: 16\% $(n=26)$ by personal inquiry and $84 \%(n=136)$ by postal dispatch. Of all chief physicians, $83 \%$ showed no interest (no response: $n=116$, active declining: $n=19)$. We presented the study to the remaining 27 chief physicians and staff (typically volunteering senior HPs), out of which 6 chose not to participate. All hospitals that declined participation were asked for reasons for non-participation, and 16 hospitals replied: Lacking resources were mentioned most frequently, followed by temporal overlap with other ongoing projects (scientific studies, or adoption of a new hospital information technology system), unsuitability of the hospital (organisation or patient population) or low expected benefit for the hospital (eg, when the established discharge procedure was perceived as similar to the study intervention).

Ultimately, 21 hospitals agreed to participate. Of these, 16 were acute and 5 rehabilitation hospitals, 2 were academic and 19 non-academic hospitals, and ward sizes ranged from 15 to 180 beds.

From the interviews with the chief physicians, we identified 13 themes; 8 corresponding to facilitators and 5 to barriers to study participation and implementation. Themes with constituent codes and example quotes are presented in table 1 . As an immediate reaction to barriers identified, we summarised potential solutions and presented them to subsequently approached hospitals. For instance, to mitigate concerns of undue effort, we recommended to involve non-medical personnel for administrative tasks and provided time estimates required for the different study steps. We also tried to target facilitators, for example, by emphasising the potential benefits for the clinics and by preparing a study announcement for the hospitals to use for information and marketing purposes.

\section{Senior HPs \\ Recruitment}

Recruitment of senior HPs was organised by the chief physicians. In total, 74 (40\% female) senior HPs participated (median 3 per hospital, IQR 2-5, min 1, $\max 9$ ). 
Table 1 Facilitators and barriers to study participation from the chief physicians' perspective

\section{Facilitators}

\begin{tabular}{ll}
\hline Themes & Codes \\
\hline Quality improvement & $\begin{array}{l}\text { Standardisation of processes } \\
\text { (discharge, medication review) }\end{array}$
\end{tabular}

Communication with GPs

\section{Quotes}

"That we get a certain standardization of the processes with these intervention tools; also when there are rotations - we have junior HP that stay for two years, then the next ones arrive - that we can integrate that in our process flows, certain tools, to standardize that." (D-01)

Communication with GPs
\[ \begin{array}{l}\text { Patient outcomes (medication, } \\ \text { hospital rehospitalisation, safety, } \\ \text { satisfaction) }\end{array} \]
Quality control
Benchmarking
"It's always a little ambivalent: on the one hand [the GPs] want to be informed, on the other hand they don't like to be called. Because they feel that they are being interrupted, and you don't really know what the best strategy is to communicate with your GP." (D-02)

"For me it's actually about patient safety." (D-03)

Validation of the hospital's strategy

$\begin{array}{ll}\text { Teaching } & \text { Teaching junior HPs } \\ & \begin{array}{l}\text { Sensitisation/ } \\ \text { awareness of HPs }\end{array} \\ \text { Scientific interest } & \text { Intrinsic interest }\end{array}$

Relevance Important topics

Challenging topics

Credibility Evidence-based approach

Ownership

$\begin{array}{ll}\text { Publicity } & \text { Individual } \\ \text { Hospital } & \text { Right time, right place } \\ \text { Fitting conditions } & \text { Target population } \\ & \begin{array}{l}\text { Complementary to ongoing } \\ \text { projects }\end{array}\end{array}$

"That it will reveal where we actually stand with our hospital, that there is also some possibility of benchmarking." (D-03)

"It is also just for us to check "is our philosophy somehow also the right one; what can we improve?'" (D-04)

"We work with many very young junior HPs, so we thought that nothing better could have happened to us than receiving such a support as your checklist." (D-05)

"My personal expectation, which I have also communicated to my senior HPs, is that we raise awareness for the discharge management, and in particular for the medication." (D-06)

"As I said, we want to do science, this is part of our job here, so that is certainly one of the key factors." (D-07)

"So in the end it is the topic that tipped the scales, it is an important topic, it is an everyday topic, it is a topic that has been studied little else, with big consequences ... that is the main point." (D-08)

"The transition of medication in the hospital to GPs is a problem that we are aware of. It is somehow a difficult interface, which we have of course already identified ourselves." (D-09)

"Many of these quality measures that are in place in hospitals today give a lot of work, and we are not sure how much they are worth... For me, it is crucial that it is studied scientifically rather than some authority coming along and saying 'now you have to do that'." (D-10)

"The fact that [the study] is run by the University Hospital Zurich also played a role for me personally." (D-11)

"It's also a bit of a flagship for me, that I brought the University of Zurich to [this hospital), along with myself, I might say. So this is my personal interest in the whole story." (D-06)

"We were published in the newspaper with too high rehospitalisation rates, and this is a tool to look at this." (D-12)

"And now that a study has just been completed, this actually fits into our sequence quite well." (D-07)

"We treat many patients who are older than 60 years (...). Many of them have many drugs. [The study] inclusion criteria are more than five; we sometimes have patients with 20 or more drugs, with proper polypharmacy." (D-11)

"And when we received the offer to participate in this study, we saw it as the perfect complement to the other projects currently underway." (D-06) 
Table 1 Continued

\begin{tabular}{ll}
\hline Barriers & Codes \\
\hline Themes & $\begin{array}{l}\text { Limited time and workforce } \\
\text { Lack of financing }\end{array}$ \\
\hline Resources & $\begin{array}{l}\text { Challenging patient population } \\
\text { (oldest old, cognitive limitations, } \\
\text { Mealth literacy) }\end{array}$
\end{tabular}

Insufficient data quality

Staff fluctuation "I'm retiring, there's a successor who doesn't know that I've

\section{Quotes}

"[It's] always the effort." (D-10)

"I mean, there is no provision for research to be carried out in clinical practice, and we are not compensated for it." (D-10)

" $99.9 \%$ of our patients do not know what 'quality of life' is. This questionnaire is complete hokum in the countryside, you can just forget about it. Because the standard answer will be: 'You tell me'." (D-06)

"Not necessarily the amount of time, but the accuracy of the work [by the junior HPs], or, in other words, whether they still achieve the same quality in intense periods, under more strain." (D-12) agreed to this... But I'll tell him. And I have now also obliged [name of a senior HP]; I told him that he has to take over, and as you have heard, there are also changes among the senior HPs." (D-08)

Intervention parts already

"Regarding the 'communication with the GPs' it is possible that the established (usual care) hurdle you are trying to overcome is not there at all in our hospital. (...) This will be difficult to evaluate." (D-12)

$\begin{array}{ll}\begin{array}{l}\text { Organisational } \\ \text { limitations }\end{array} & \begin{array}{l}\text { Technical limitations/information } \\ \text { technology }\end{array}\end{array}$

"At first glance, it all sounds simple, but we saw for ourselves, you were there too, there were already a few hurdles where we simply had to think about a few things, how to do that, the hospital information system is of course not the same everywhere, but these are more the technical and organizational things." (D-08)

Integration in clinical routine/ "Whether it can be sensibly implemented in everyday clinical paper-based data collection practice; that was certainly a topic of discussion." (D-10)

Motivation of staff Missing motivation/scepticism

"I think the only hurdle we have to face now is, of course, that the junior HPs, who already have a large workload, must now be motivated and convinced that this is a good thing that it's worth investing time for now." (D-05)

$\begin{array}{lll}\text { Relationship with GPs } \quad \text { Concern of bypassing GPs } & \text { "I think it is important - because we are in very close contact } \\ & \text { with the GPs - that [the GPs] will not get the feeling that we are } \\ & \text { participating in a study with their patients and [the GPs] might not } \\ & \text { have wanted that." (D-05) }\end{array}$

Themes identified from the interviews $(n=15)$, with corresponding codes and example quotes from chief physicians (anonymised).

GP, general practitioner; HP, hospital physician.

The median work experience was 15 years (IQR 10-24), not significantly different between study arms $(p=0.971)$. Of the 60 senior HPs responding to the initial paper-based survey, $23 \%(n=14)$ had experience with scientific studies, and $35 \%(n=21)$ had been involved in a project or study regarding related topics (polypharmacy/appropriate medication, frailty, discharge management or communication with GPs). All but three of the responding senior HPs found the topic of the study very relevant or relevant to them. Motivation for participation in the study were most frequently quality improvement $(45 \%, n=27)$ and relevance of the topic $(28 \%, n=17)$, but $23 \%(n=14)$ of senior HPs stated that they had no motivation or that it was the chief physician's decision. The most frequently mentioned concerns were methodological limitations regarding recruitment, follow-up or risk of bias $(22 \%, n$ $=13$ ) and missing resources/high workload $(8 \%, n=5)$, but the majority of senior HPs $(62 \%, n=37)$ mentioned no concerns.

\section{Delivery}

The intervention was delivered to senior HPs in terms of an instruction by the study team (see the Design and setting subsection under the Methods section). The instruction was performed uniformly by the principal investigator using presentation slides.

\section{Response}

All but one of the senior HPs stated that the instruction had met their expectations. The study aim, the study flow in the hospitals and their role in the study were very clear or clear to all but three senior HPs.

The senior HPs' attitudes and responses to the checklist and adaptation of the discharge letter as declared 
A) General attitudes

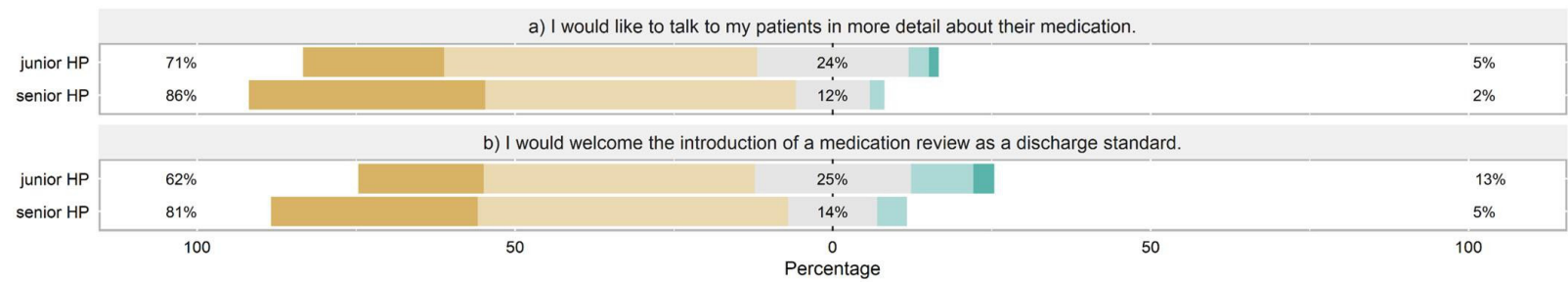

B) Checklist

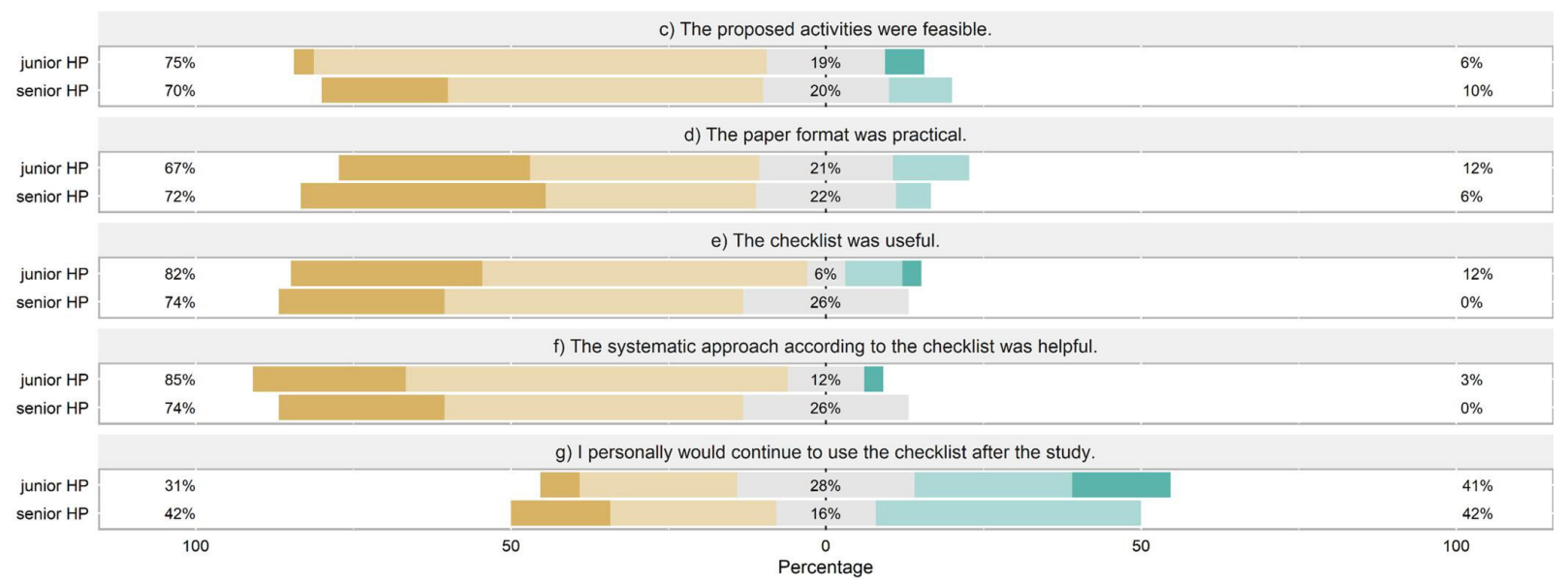

C) Discharge letter

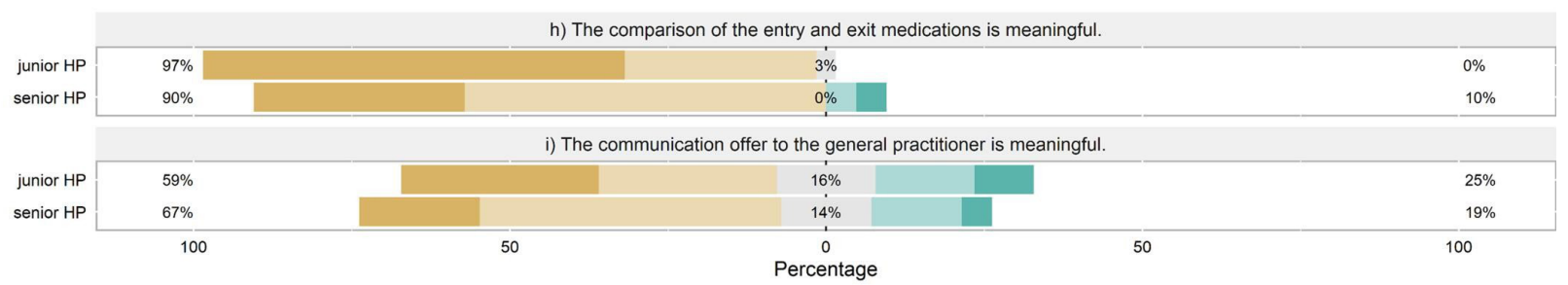

fully applies largely applies partially applies d does rather not apply does not apply at all

Figure 3 Attitudes and perception of feasibility and usefulness of tools and procedures by junior and senior hospital physicians (HPs). Questions about general attitudes (A) were answered by HPs in both study arms (senior HPs: $n=44$, junior HPs: $n=65)$; questions regarding checklist $(B)$ and discharge summary (C) were only directed at the intervention group (senior HPs: $n=24$, junior HPs: $n=38$ ). The percentages given indicate (1) largely applies or applies, (2) partially applies, (3) does rather not or not apply.

in the online survey at the end of the study are shown in figure 3A-C, respectively. The majority of senior HPs appreciated the relevance of the topic and perceived the intervention as feasible and helpful. For example, respondents stated that the checklist reminded them to review drugs more critically $(46 \%, n=11)$, to consistently motivate patients to visit their GPs within 7 days $(38 \%, n=9)$ or to discuss treatment goals with the patients $(17 \%, n$ $=4)$. Fewer $(42 \%)$ senior HPs declared that they would continue using the checklist after the study (figure 3B). Two senior HPs suggested that the checklist could be improved by choosing the time period from hospital discharge to GP visit individually for each patient (instead of 7 days as required by the checklist).

To the five questions regarding (de)prescribing decisions (case vignettes, see online supplemental appendix $2), 69 \%(n=69)$ of responses in the intervention group were pro-deprescribing (reducing/stopping or switching to phytotherapeutics, vs continuing/increasing), while in the control group, the corresponding proportion was $71 \%(n=71, p=0.877)$.

\section{Junior HPs}

\section{Recruitment}

Frequent rotations of junior HPs within and between hospitals necessitated their recruitment and instruction by senior HPs rather than the study team. Their exact recruitment number is therefore unknown, but 164 junior HPs were ultimately involved in intervention delivery ( $54 \%$ female), with a median of 6 (IQR 4-10, min 1, max 28) per hospital. Their median work experience was 2 years (IQR 1-4, min 1, max 10), with no significant difference between study arms $(p=0.590)$. 


\section{Delivery}

The junior HPs' instruction was either incorporated into their mandatory continuing education, performed in a dedicated meeting for groups of junior HPs, or by means of a one-to-one instruction. In most hospitals, a mix of formats was applied. In total, senior HPs spent a median of $45 \mathrm{~min}$ (IQR 18-60) to deliver the theory part of the instruction (intervention group); individual junior HPs were instructed for a median of $15 \mathrm{~min}$ (IQR 10-30). To improve delivery to junior HPs, we provided the senior HPs with presentation slides covering both theory and data handling, and distributed practice material, summaries with key information and extensive information leaflets for junior HPs.

\section{Response}

The junior HPs' attitudes and response to the intervention are shown in figure 3A-C. The checklist and the adaptations to the discharge letter were rated feasible and helpful by the majority of junior HPs (figure 3B,C). Fifty percent $(n=19)$ stated that they were reminded to question each drug in the patients' medication regimes more rigorously, $45 \%(n=17)$ felt stimulated to motivate the patients to visit their GP after discharge and 21\% ( $n$ $=8$ ) to discuss treatment goals with their patients. Only a minority $(31 \%, n=10)$ intended to continue using the checklist after the study (figure 3B).

For the five medication review questions in the case vignettes, in the intervention group, deprescribing was suggested in $70 \%(n=111)$ of responses versus $59 \%(n=$ $68)$ in the control group $(p=0.103)$.

\section{Patients}

\section{Recruitment and reach}

Patients were recruited at admission to the ward by the participating HPs on duty. The total number of recruited patients was 612 (50\% in the intervention group), with a median of 21 patients (IQR 15-37, min 8, max 91) per hospital. To facilitate recruitment, we provided the hospitals with a disposable information sheet for patients, and a condensed version to be used for verbal clarification when recruiting patients. In the digital survey at the end of the study, most HPs stated that the short statement was used always $(65 \%, n=66)$ or sometimes $(25 \%, n=25)$ when recruiting patients.

\section{Delivery}

Depending on the hospital, junior and/or senior HPs carried out the discharge procedure on the patient. In median, each senior HP completed two checklists (IQR 0-10, min 0, max 20), while junior HPs filled out a median of five checklists (IQR 4-8, min 1, max 25). The HPs declared that checklist activities were begun during the patients' hospital stays-as opposed to shortly before discharge-for the majority of patients (median over HPs $61 \%$, IQR 33\%-86\%, min 0\%, $\max 100 \%$ ).

Intervention delivery to patients (in intervention hospitals) was high: The proposed activities were reportedly carried out in $95 \%$ ( $n=3766$ ticks on the 14 -item checklists). All but three checklist items had been ticked in over $90 \%$, and the lowest execution rate $(83 \%)$ was reported for 'motivating patients to consult their GP within 7 days'.

\section{Response}

According to half of the HPs in the intervention group responding to the specific question in the digital survey $(n=51)$, patients appreciated being involved in decisions regarding their medication plans, and only $7 \%$ of the patients (median over senior and junior HPs; IQR 0\%-25\%, min 0\%, max 60\%) rejected proposed changes to their medication plans. Common reasons for patients' resistance to medication changes were habits/being used to a specific drug, believing in its positive effect, loyalty to the GP who prescribed the drug or a general resistance to change. Some HPs additionally mentioned that the patients' addiction to the medication (eg, to benzodiazepines or opioids) or communication barriers (language) impeded changing the medication plan.

During follow-up, the majority of patients did not return the required documents in time and had to be reminded by phone call. The overall loss to follow-up rate within the first month after discharge was $31 \%$ ( $n=194$ patients, see figure 2). Most frequent reasons for loss to follow-up were inability or unwillingness to return the requested documents. Patients mentioned being too sick or old to fill out the questionnaire, lack of motivation/perceived benefit, or previous unawareness of the questionnaire. Loss to follow-up rates varied between hospitals (median $31 \%$, IQR 26\%-38\%, $\min 9 \%, \max 55 \%$ ).

\section{GPs}

\section{Delivery}

The intervention was delivered to GPs indirectly via adaptations to the discharge letter, that is, the reorganised presentation of the patients' medications, and the communication offer to discuss medication changes with the HPs. The communication offer, as a fixed component of the intervention, was quantitatively well implemented (in rare cases only added after an early reminder) but often inserted very inconspicuously at the end of the discharge letter. For 22\% of patients in the intervention hospitals and 18\% in control hospitals, the GPs were contacted by HPs during the hospital stay already. Regarding the presentation of the patients' medication in the discharge letter, a flexible implementation approach was required, mainly due to the rigidity of the hospitals' clinical information systems. Three modes of implementations were accepted, with decreasing preference (number of hospitals which chose the option is indicated in brackets): (1) dedicated table of medication changes, with reasons for each change $(n=1)$; (2) separate tables of admission and discharge medication, adjacent or in immediate sequence, again with explanations of medication changes $(n=8)$; (3) table of discharge medication only, with changes explained in the text body $(n=3)$. Inspection of the discharge letters revealed that 


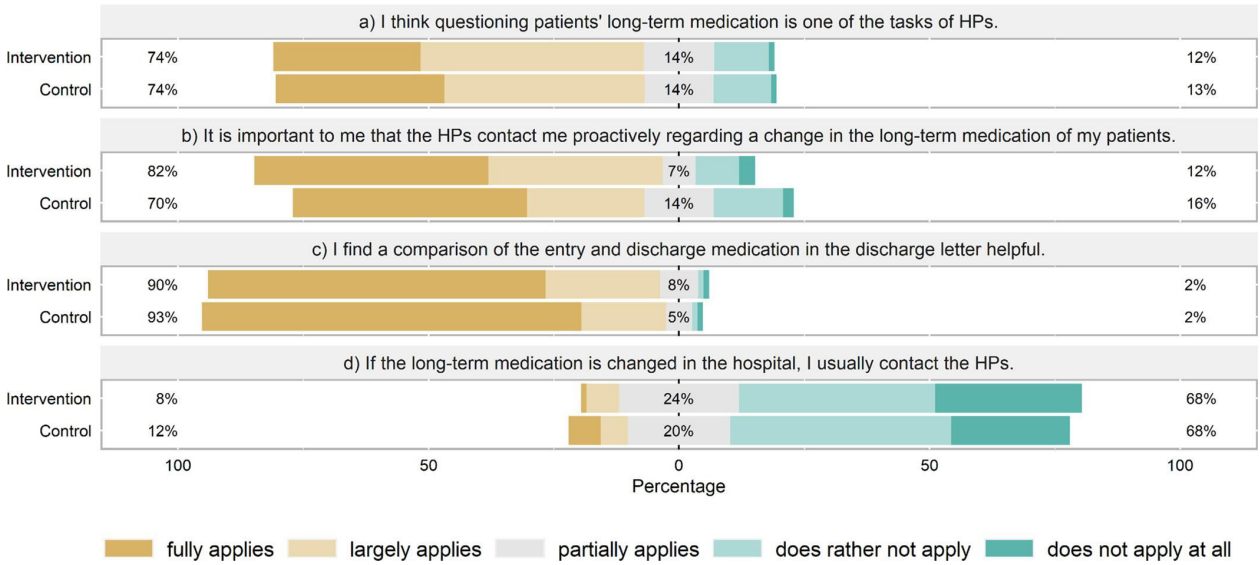

Figure 4 General practitioners' (GPs) views on medication review and communication at hospital discharge. The percentages given indicate (1) largely applies or applies, (2) partially applies, (3) does rather not or not apply (GPs: $n=187$ ). HP, hospital physicians.

medication changes were often incompletely explained, irrespective of the presentation mode.

\section{Response}

Of the GPs responding to the postal survey $(n=187)$, the vast majority considered a comparison of admission and discharge medication in the discharge letter helpful $(91 \%$, figure 4$)$. Most also agreed that HPs should review the long-term medication of patients $(74 \%)$ and appreciated being contacted in case of medication alterations $(76 \%)$. Only few GPs (10\%) would contact the HPs themselves when noticing a change. In the absence of contact, most GPs (74\%) declared to usually-but depending on the individual case-adopt changes to the long-term medication made by HPs. Many GPs stated that an explanation for modifying/altering the medication was very important. Another issue raised by many GPs was that switching between original and generic drugs could confuse patients and entail the risk of double intake. They proposed that the medication should be reset to preparations used at admission or at least that patients should be informed.

Documenting GP-initiated contacts with HPs following patient discharge was in the responsibility of HPs who reported 14 contacts in total.

\section{Maintenance}

The median patient inclusion period per hospital was 205 days (IQR 168-271 days, min 23, max 325), corresponding to approximately 7 months per hospital. Inclusion intensity varied over time and among hospitals and was not noticeably influenced by roughly monthly newsletters (online supplemental appendix 3). The designated contact persons in the hospitals (study nurse, clinical trials unit, senior HP or chief physician) were reminded by email and phone if patient recruitment was still low. Not only recruitment yield, but also immediate loss to follow-up rates changed over time in some hospitals. Regarding qualitative aspects of study delivery, when asked in the online survey whether their discharge management had changed over the course of the study, $15 \%(n=14)$ of the responding HPs agreed, declaring, for instance, that they had reviewed drugs more carefully or earlier, had explained them more carefully to the patients and that their contact with GPs had intensified.

\section{Context}

Swiss healthcare setting

Switzerland is organised as a federalist system of 26 cantons enjoying a high degree of autonomy vis-à-vis the federal government. ${ }^{23}$ The federalist organisation of healthcare results in regulated competition between hospitals and high variability, for example, in clinic information systems used by hospitals. ${ }^{24}$ While hospitals mandatorily use digital patient records, this is not the case for ambulatory physicians including GPs. In fact, digitalisation in the ambulatory healthcare sector in Switzerland is rather low. In 2018, only $43 \%$ of GPs documented their patient records fully electronically. ${ }^{25}$ The fragmented digitalisation of ambulatory healthcare likely hinders effective communication between hospitals and GPs.

\section{COVID-19}

The COVID-19 pandemic had serious implications for this hybrid trial. The novel virus hit Switzerland in early March 2020, whereupon hospitals were ordered to stop all elective surgeries and ongoing trials. At that time point, the study was still ongoing in 13 (of the 21) hospitals, of which 10 had to stop and 3 to postpone recruitment, thus limiting the study sample and delaying completion of the study.

\section{DISCUSSION}

This process evaluation provides insights into the implementation of a cluster RCT set at the interface between hospital care and general practice. Using a tailored version of the well-established framework by Grant $e t a l^{16}$ for process evaluations of complex multilevel interventions, 
increased knowledge about the trial's implementation on different levels was gained.

Recruitment of hospitals was laborious but successful. Minimal workload and a perceived benefit for the clinic proved to be crucial for participation. Intervention dose was high, but intervention fidelity was limited (adaptations to discharge letter) or unknown (medication review). Recruitment and retention of patients was challenging, partly due to patient characteristics (old, frail) and the COVID-19 pandemic. The intervention was deemed feasible and helpful by HPs, and the relevance of the topic appreciated by both HPs and GPs.

\section{Hospital level}

As expected, it was challenging to recruit hospitals that face market competition to participate in an external study without providing financial incentives. In addition to barriers related to resources, chief physicians mentioned concerns regarding methodological or organisational limitations (table 1) and motivation of HPs. The majority of these issues have been reported before in a systematic review exploring barriers towards the implementation of hospital-based interventions. ${ }^{26}$ As for facilitators, we found that a perceived benefit to the clinic or chief physician was crucial.

\section{HP level}

Most senior HPs showed motivation to participate in the study, for example, because of expected quality improvements or in recognition of the topic's relevance. Almost one out of four, however, stated that they only participated following the hospital/chief physician's decision. This is problematic, as lack of motivation is a well-known barrier towards implementation. ${ }^{26}$ To increase motivation, Geerligs $e t a l^{26}$ suggest to share informal intervention 'success stories'. In our case, these could be examples of patients with successfully improved medication lists, or a positive communication experience with a GP. Involving senior HPs earlier in the study design might further benefit the study by stimulating an essential sense of ownership. ${ }^{26}$

To capture the change in knowledge and attitudes of HPs towards deprescribing (a proxy for the expected training effect in our intervention model), we relied on case vignettes. Readiness to deprescribe in defined patient conditions was not significantly different between intervention and control groups (which might partly be attributable to methodical limitations, see the Strengths and limitations section). However, to capture the positive impact of an intervention, it is also important to take views and experiences of staff into account. ${ }^{12}$ The HPs' feedback to the intervention was positive: The adaptations of the discharge letter, especially the comparison of discharge with admission medication, were welcomed not only by senior and junior HPs but also by GPs (see the GP level section). This is particularly interesting as it could be implemented in clinical information systems without increasing the HPs' workload. Regarding the checklist, the majority of HPs stated that the proposed activities were feasible, and that the checklist was useful. Nonetheless, only a minority (one out of three) of junior and senior HPs intended to use the checklist after the study. The reasons for this are unclear. We can only speculate that additional time need or costs must have exceeded the expected benefits. ${ }^{27}$ Further exploration of potential reasons for this reluctance would be needed before scaling up the dissemination of our checklist.

\section{Patient level}

This intervention was targeted at multimorbid patients over 60 years of age. It is well known that recruiting and retaining old, multimorbid, frail and cognitively impaired patients is challenging. ${ }^{12}$ Hence, old and frail patients are often excluded from trials, ${ }^{28}$ even though they might profit most from interventions regarding medication review or communication. Recruiting frail or cognitively impaired patients was encouraged within this study and accordingly brought along some difficulties. For instance, the detailed information forms overwhelmed most patients. The short statement we provided to HPs was partially useful to overcome this barrier. However, the reluctance of vulnerable and multimorbid patients to take on another task (the one of study participation) besides their high burden of disease remained a major challenge. This is mirrored by the fact that the final number of recruited patients was substantially lower than expected, even taking the COVID-19 pandemic-related barriers towards recruitment into account.

Judging by the checklist ticks, the intervention was well delivered to the patients in terms of quantity (dose), but we were unable to evaluate delivery quality (fidelity), that is, to what extent HPs involved the patients and what effort they made in reviewing the patients' medication.

We assessed the patients' acceptance of our approach only indirectly via HPs. HPs declared that approximately half the patients appreciated being involved in decisions regarding their medication plans, and only very few rejected the proposed changes with reasons similar to those identified in recent qualitative studies with older adults and their carers. ${ }^{19} 29$ Interestingly, a recent systematic review detected a lack of family involvement in managing medications of older patients across transitions of care, ${ }^{30}$ an aspect that might merit further investigation.

The variability of loss to follow-up rates and reasons for loss to follow-up between and even within hospitals suggested that quality of patient information (particularly regarding the patients' post-discharge responsibilities) and the type of recruited patient population (i.e., proportion of patients with cognitive impairment or with a high number of diseases) varied among hospitals and HPs. Many patients were unable or unwilling to fill out the required questionnaires during follow-up. Lyles $e t a l^{31}$ suggested that remuneration of participants in recognition of their time commitment and a consistent, clear and persistent communication with participants were important factors in enrolling and retaining subjects. 


\section{GP level}

Similar to other researchers of routine care, ${ }^{32}$ we faced the dilemma inherent to any flexible implementation approach: Allowing high flexibility to suit the local circumstances may increase recruitment chances while decreasing implementation fidelity. We gave the hospitals much freedom in the intervention delivery to GPs, in particular regarding the adaptations of the discharge letter, which resulted in suboptimal implementation fidelity. The contact offer was presented very inconspicuously in several hospitals, and the medication changes were often not properly explained.

The high response rate of GPs contacted by postal dispatch and their feedback on the relevance of the topic indicated the need for better discharge protocols, thus justifying our trial. Accordingly, much literature is available on this topic. For instance, several studies from different countries reported that GPs appreciated receiving information on medication changes and reasons in the discharge letter. ${ }^{33-36}$ Our findings show that GPs perceived this as more convenient than having to actively call the-often unavailable or difficult to contactHPs. Therefore, and not too surprisingly so, the number of GPs contacting HPs after their patients' discharge was low. However, the number was so exceptionally low that we must also assume incomplete documentation by HPs. The finding that most GPs reportedly adopted changes introduced by HPs is in accordance with a Danish qualitative study which concluded that the poor continuity of medication changes at sector transition was not due to the GPs' deliberate actions of removing the patients' medications, but presumably due to procedural errors in the follow-up on the patient after discharge instead. ${ }^{37}$

\section{Strengths and limitations}

We used both quantitative and qualitative approaches to achieve an adequate insight into the implementation of the intervention as proposed by the UK Medical Research Council guidelines. ${ }^{1138}$ Moreover, we performed the evaluation before instead of alongside or after the analysis and publication of the main findings, which, to our knowledge, is an exception and an innovative approach for process evaluations and ensures that the evaluation is blind to (and thus not biased by) trial outcomes.

The practical and well-structured framework by Grant $e t$ $a l^{16}$ proved very useful in conducting this multilevel process evaluation. It has already been applied in numerous process evaluations of cluster RCTs ${ }^{32}{ }^{39-43}$ but was often reduced to specific elements. ${ }^{32}$ 38-40 43 While Roberts $e t$ a $t^{42}$ claimed to be the first to use the framework in its entirety for a process evaluation of a cluster RCT, we used an even more extensive version adapted to our intervention: The framework was extended with additional levels for the intermediary providers (junior HP) and the overarching institutions (hospitals). This comprehensive approach allowed us to study every level of the intervention delivery and response. Following our example, the framework could be further extended to handle any number of clustering levels.

We described many implementation support strategies. Future trials on hospital discharge optimisation may benefit from information on barriers to be tackled and facilitators to be taken into account on every level of the intervention. This may ultimately contribute to narrow the gap between the evidence of such strategies and their application in routine care.

Limitations of the study were the small sample of HPs, and that non-responders potentially introduced a selection bias (volunteer bias) to the digital survey. The low response rate of junior HPs was partially due to the frequent rotations; many junior HPs were no longer working at the hospital at the time of the process evaluation (which we anticipated). Furthermore, there might have been some desirability bias in the answers of HPs and chief physicians. We also faced potential bias due to unblind chief physicians and senior HPs, which was inevitable during the hospital recruitment process. Lastly, it is conceivable that some selection bias was introduced by HPs recruiting fitter patients, even though we tried to mitigate this.

\section{CONCLUSION}

The process evaluation framework by Grant $e t a l^{16}$ proved helpful for investigating the implementation of a complex and multifaceted intervention at different levels in a hospital setting. Our approach can be tailored and adapted to similar interventions. The results from this evaluation will support interpretation of the findings of the effectiveness study and-positive results given-dissemination of our approach to further hospitals. In addition, the barriers and facilitators, as well as targeted implementation strategies presented may help researchers and policy makers to plan and implement future studies and quality improvement programmes in the hospital setting.

Acknowledgements We thank Susan Groth (our study nurse), Petra Hug-Gresens, and all physicians and patients who directly or indirectly provided data for this study, for their invaluable contributions to the study.

Contributors YR, TG and SN-J contributed to conceptualisation, investigation and methodology. YR performed the formal analysis and visualisation and wrote the original draft. TG was responsible for project administration and contributed to the original draft. SN-J was responsible for funding acquisition and supervision. YR, TG and $\mathrm{SN}-\mathrm{J}$ reviewed, edited and approved the final version of the manuscript.

Funding This work was funded as part of the National Research Programme 'Smarter Health Care' (NRP74) by the Swiss National Science Foundation (grant no. 407440_167193, 7 December 2016). Additional funding was provided by the University of Zurich and the University Hospital Zurich.

Competing interests None declared.

Patient consent for publication Not required.

Ethics approval Ethical approval was obtained from the Ethics committee of the Canton of Zurich (as part of the full effectiveness-implementation trial, BASEC-No. 2018-00215). Informed consent was obtained from all individual participants included in the study.

Provenance and peer review Not commissioned; externally peer reviewed.

Data availability statement Data are available on reasonable request. The datasets used and/or analysed during the current study are available from the last author (SN-J) on reasonable request.

Supplemental material This content has been supplied by the author(s). It has not been vetted by BMJ Publishing Group Limited (BMJ) and may not have been peer-reviewed. Any opinions or recommendations discussed are solely those of the author(s) and are not endorsed by BMJ. BMJ disclaims all liability and 
responsibility arising from any reliance placed on the content. Where the content includes any translated material, BMJ does not warrant the accuracy and reliability of the translations (including but not limited to local regulations, clinical guidelines, terminology, drug names and drug dosages), and is not responsible for any error and/or omissions arising from translation and adaptation or otherwise.

Open access This is an open access article distributed in accordance with the Creative Commons Attribution Non Commercial (CC BY-NC 4.0) license, which permits others to distribute, remix, adapt, build upon this work non-commercially, and license their derivative works on different terms, provided the original work is properly cited, appropriate credit is given, any changes made indicated, and the use is non-commercial. See: http://creativecommons.org/licenses/by-nc/4.0/.

\section{ORCID iDs}

Yael Rachamin http://orcid.org/0000-0002-1484-6934

Thomas Grischott http://orcid.org/0000-0002-1564-5296

Stefan Neuner-Jehle http://orcid.org/000-0002-6260-8148

\section{REFERENCES}

1 Maher RL, Hanlon J, Haijar ER. Clinical consequences of polypharmacy in elderly. Expert Opin Drug Saf 2014;13:57-65.

2 Calderón-Larrañaga A, Poblador-Plou B, González-Rubio F, et al. Multimorbidity, polypharmacy, referrals, and adverse drug events: are we doing things well? Br J Gen Pract 2012;62:e821-6.

3 Sganga F, Landi F, Ruggiero C, et al. Polypharmacy and health outcomes among older adults discharged from Hospital: results from the crime study. Geriatr Gerontol Int 2015;15:141-6.

4 Frazier SC. Health outcomes and polypharmacy in elderly individuals: an integrated literature review. J Gerontol Nurs 2005;31:4-11.

5 Nobili A, Licata G, Salerno F, et al. Polypharmacy, length of hospital stay, and in-hospital mortality among elderly patients in internal medicine wards. The REPOSI study. Eur J Clin Pharmacol 2011:67:507-19.

6 Cullinan S, Raae Hansen C, Byrne S, et al. Challenges of deprescribing in the multimorbid patient. Eur $J$ Hosp Pharm 2017:24:43-6.

7 Rankin A, Cadogan CA, Patterson SM, et al. Interventions to improve the appropriate use of polypharmacy for older people. Cochrane Database Syst Rev 2018;9:Cd008165.

8 Gonçalves-Bradley DC, Lannin NA, Clemson LM, et al. Discharge planning from hospital. Cochrane Database Syst Rev 2016;23:Cd000313.

9 Grischott T, Zechmann S, Rachamin Y, et al. Improving inappropriate medication and information transfer at hospital discharge: study protocol for a cluster RCT. Implement Sci 2018;13:155.

10 Oakley A, Strange V, Bonell C, et al. Process evaluation in randomised controlled trials of complex interventions. $B M J$ 2006;332:413-6.

11 Craig P, Dieppe P, Macintyre S, et al. Developing and evaluating complex interventions: the new medical Research Council guidance. BMJ 2008;337:a1655-a55.

12 Datta J, Petticrew M. Challenges to evaluating complex interventions: a content analysis of published papers. BMC Public Health 2013;13:568.

13 Moore GF, Audrey S, Barker M, et al. Process evaluation of complex interventions: medical Research Council guidance. BMJ 2015;350:h1258.

14 Campbell M, Fitzpatrick R, Haines A, et al. Framework for design and evaluation of complex interventions to improve health. $B M J$ 2000;321:694-6.

15 Carroll C, Patterson M, Wood S, et al. A conceptual framework for implementation fidelity. Implement Sci 2007;2:40.

16 Grant A, Treweek S, Dreischulte T, et al. Process evaluations for cluster-randomised trials of complex interventions: a proposed framework for design and reporting. Trials 2013;14:15.

17 Landes SJ, McBain SA, Curran GM. An introduction to effectivenessimplementation hybrid designs. Psychiatry Res 2019;280:112513.

18 Cooper JA, Cadogan CA, Patterson SM, et al. Interventions to improve the appropriate use of polypharmacy in older people: a Cochrane systematic review. BMJ Open 2015;5:e009235.

19 Zechmann S, Trueb C, Valeri F, et al. Barriers and enablers for deprescribing among older, multimorbid patients with polypharmacy: an explorative study from Switzerland. BMC Fam Pract 2019;20:64.

20 Pinnock H, Barwick M, Carpenter CR, et al. Standards for reporting implementation studies (STARI) statement. BMJ 2017;356:i6795.

$21 \mathrm{R}$ Core Team. R: a language and environment for statistical computing. Vienna, Austria: R Foundation for Statistical Computing, 2018.
22 Braun V, Clarke V, et al. Thematic analysis. In: Cooper H, Camic PM, Long DL, eds. APA Handbook of research methods in psychology. Washington, DC: American Psychological Association, 2012: 57-71.

23 Federal Department of Foreign Affairs. The cantons, 2020. Available: https://www.eda.admin.ch/aboutswitzerland/en/home/politik/ uebersicht/kantone.html\#: :text=Switzerland\%20is\%20divided\% 20into\%2026,culture\%2C\%20religion\%20and\%20socioeconomic\% 20structure

24 PricewaterhouseCoopers. [The hospital information system of the future], 2017. Available: https://www.pwc.ch/de/publications/2017/ blog_kisderzukunft_de-1.pdf

25 Federal Statistical Office. Doctors' surgeries and out-patient centres: structural data for 2018 Neuchâtel, 2020. Available: https:// www.bfs.admin.ch/bfs/en/home/news/whats-new.gnpdetail.20200152.html

26 Geerligs L, Rankin NM, Shepherd HL, et al. Hospital-Based interventions: a systematic review of staff-reported barriers and facilitators to implementation processes. Implement Sci 2018;13:36.

27 Redmond P, Munir K, Alabi O, et al. Barriers and facilitators of medicines reconciliation at transitions of care in Ireland - a qualitative study. BMC Fam Pract 2020;21:116.

28 Boyd CM, Vollenweider D, Puhan MA. Informing evidence-based decision-making for patients with comorbidity: availability of necessary information in clinical trials for chronic diseases. PLOS One 2012; 7:e41601.

29 Reeve E, Low L-F, Hilmer SN. Beliefs and attitudes of older adults and carers about deprescribing of medications: a qualitative focus group study. Br J Gen Pract 2016;66:e552-60.

30 Manias E, Bucknall T, Hughes C, et al. Family involvement in managing medications of older patients across transitions of care: a systematic review. BMC Geriatr 2019;19:95.

31 Lyles JS, Hodges A, Collins C, et al. Using nurse practitioners to implement an intervention in primary care for high-utilizing patients with medically unexplained symptoms. Gen Hosp Psychiatry 2003;25:63-73.

32 Mann C, Shaw ARG, Guthrie B, et al. Can implementation failure or intervention failure explain the result of the $3 \mathrm{D}$ multimorbidity trial in general practice: mixed-methods process evaluation. BMJ Open 2019;9:e031438.

33 Adam H, Niebling W-B, Schott G. [The information about discharge medication: what do general practitioners need?]. Dtsch Med Wochenschr 2015;140:e74-9.

34 Weetman K, Dale J, Spencer R, et al. Gp perspectives on hospital discharge letters: an interview and focus group study. BJGP Open 2020;4. doi:10.3399/bjgpopen20X101031. [Epub ahead of print: 23 06 2020].

35 Caleres G, Strandberg EL, Bondesson Åsa, et al. Drugs, distrust and dialogue -A focus group study with Swedish GPs on discharge summary use in primary care. BMC Fam Pract 2018;19:127.

36 Spencer RA, Rodgers S, Salema N, et al. Processing discharge summaries in general practice: a qualitative interview study with GPs and practice managers. BJGP Open 2019;3:bjgpopen18X101625.

37 Strehlau AG, Larsen MD, Søndergaard J, et al. General practitioners' continuation and acceptance of medication changes at sectorial transitions of geriatric patients - a qualitative interview study. BMC Fam Pract 2018;19:168.

38 Brady MC, Stott DJ, Norrie J, et al. Developing and evaluating the implementation of a complex intervention: using mixed methods to inform the design of a randomised controlled trial of an oral healthcare intervention after stroke. Trials 2011:12:168.

39 Klingshirn $\mathrm{H}$, Müller M, Beutner K, et al. Implementation of a complex intervention to improve participation in older people with joint contractures living in nursing homes: a process evaluation of a cluster-randomised pilot trial. BMC Geriatr 2020;20:270.

40 Holle D, Teupen S, Graf R, et al. Process evaluation of the response of nursing homes to the implementation of the dementia-specific case conference concept WELCOME-IdA: a qualitative study. BMC Nurs 2020;19:14.

41 Roberts JL, Pritchard AW, Williams M, et al. Mixed methods process evaluation of an enhanced community-based rehabilitation intervention for elderly patients with hip fracture. BMJ Open 2018;8:e021486.

42 Roberts S, Mclnnes E, Bucknall T, et al. Process evaluation of a cluster-randomised trial testing a pressure ulcer prevention care bundle: a mixed-methods study. Implement Sci 2017;12:18.

43 Stephens TJ, Peden CJ, Pearse RM, et al. Improving care at scale: process evaluation of a multi-component quality improvement intervention to reduce mortality after emergency abdominal surgery (epoch trial). Implement Sci 2018;13:142. 